\title{
Europäische Einigung: Das Lissabon-Urteil und die Abwahl des Grundgesetzes
}

Gut zwei Jahre sind vergangen seit das Bundesverfassungsgericht in Fortsetzung der Maastricht-Rechtsprechung am 30.6.2009 sein Urteil zum EU-Reformvertrag, ${ }^{1}$ dem sog. Vertrag von Lissabon, verkündet hat. Wie nicht anders zu erwarten, löste diese Verkündung eine Vielzahl von streitbarem Schrifttum zu Inhalt und Konsequenzen des Urteils aus. ${ }^{2}$ Der vorliegende Beitrag bietet keine erneute Auseinandersetzung mit dem Urteil in seiner durchaus stolzen Gänze, sondern betrachtet, auch vor dem Hintergrund der derzeitigen politischen Diskussionen um die Zukunft der Europäischen Union in Krisenzeiten, den durch das Bundesverfassungsgericht festgelegten Modus ${ }^{3}$ für eine mögliche, über den derzeitigen Integrationsstand hinausgehende Weiterentwicklung der Europäischen Union (EU): die Abwahl des Grundgesetzes zugunsten einer neuen Verfassung. Hierzu geht er zunächst auf die Herleitung und Argumentation des Bundes-

1 BVerfGE 123, $267 \mathrm{ff}$.

2 Vgl. nur inter alia $R$. Bieber, An Association of Sovereign States, in: EuConst 5 (2009), S. 391 ff.; E. Denninger, Identität versus Integration?, in: JZ 2010, S. 969 ff.; K. F. Gärditz/C. Hillgruber, Volkssouveränität und Demokratie ernst genommen - Zum Lissabon-Urteil des BVerfG, in: JZ 2009, S. 872 ff.; D. Grimm, Grundgesetz als Riegel vor einer Verstaatlichung der Europäischen Union, in: Der Staat 48 (2009), S. 475 ff.; D. Halberstam/C. Möllers, The German Constitutional Court says „Ja zu Deutschland!“, in: German Law Journal 10 (2009), S. 1241 ff.; P. Häberle, Das retrospektive Lissabon-Urteil als versteinernde Maastricht II-Entscheidung, in: Jahrbuch des öffentlichen Rechts der Gegenwart, Bd. 58 (2010), S. 317 ff.; $M$. Jestaedt, Warum in die Ferne schweifen, wenn der Maßstab liegt so nah? - Verfassungshandwerkliche Anfragen an das Lissabon-Urteil des BVerfG, in: Der Staat 48 (2009), S. 497 ff.; M. Kottmann/C. Wohlfahrt, Der gespaltene Wächter? - Demokratie, Verfassungsidentität und Integrationsverantwortung im Lissabon-Urteil, in: ZaöRV, 69 (2009), S. 443 ff.; C. Ohler, Herrschaft, Legitimation und Recht in der Europäischen Union - Anmerkungen zum LissabonUrteil des BVerfG, in: AöR 135 (2010), S. 135 ff.; M. Ruffert, An den Grenzen des Integrationsverfassungsrechts: Das Urteil des Bundesverfassungsgerichts zum Vertrag von Lissabon, in: DVB1. 2009, S. 1197 ff.; C. Schönberger, Die Europäische Union zwischen „Demokratiedefizit" und Bundesstaatsverbot - Anmerkungen zum Lissabon-Urteil des Bundesverfassungsgerichts, in: Der Staat 48 (2009), S. 535 ff.; F. Schorkopf, The European Union as An Association of Sovereign States: Karlsruhes's Ruling on the Treaty of Lisbon, in: German Law Journal, 10 (2009), S. 1219 ff.; J. P. Terhechte, Souveränität, Dynamik und Integration - making up the rules as we go along? - Anmerkungen zum Lissabon-Urteil des Bundesverfassungsgerichts, in: EuZW 2009, S. 724 ff.; C. Tomuschat, Lisbon - Terminal of the European Integration Process? - The Judgement of the German Constitutional Court of 30 June 2003, in: ZaöRV 70 (2010), S. 251 ff.; D. Thym, Europäische Integration im Schatten souveräner Staatlichkeit - Anmerkungen zum Lissabon-Urteil des Bundesverfassungsgerichts, in: Der Staat 48 (2009), S. 559 ff.; R. Wahl, Die Schwebelage im Verhältnis von Europäischer Union und Mitgliedstaaten - Zum Lissabon-Urteil des Bundesverfassungsgerichts, in: Der Staat 48 (2009), S. $587 \mathrm{ff}$; $M$. Wendel, Lisbon before the Courts: Comparative Perspectives, in: EuConst 7 (2011), S. $96 \mathrm{ff}$.

3 Wissend, dass der Begriff „Modus“, wie sich noch im Verlauf des Aufsatzes zeigen wird, ein grundsätzlich zu konkreter Begriff für die Andeutungen des Urteilstextes darstellt. 
verfassungsgerichts bezüglich der Notwendigkeit einer Abwahl des Grundgesetzes für eine weitere Integration hin zu einem „Europäischen Bundesstaat“ ein (I.), bevor er die Unumgänglichkeit dieser Argumentation hinterfragt (II.) und sie in einem Vergleich den Diskussionen im Zuge der deutschen Wiedervereinigung gegenüberstellt (III.). ${ }^{4}$ Schließlich ist - bei aller Unsicherheit - vor dem Hintergrund des Dargestellten ein Ausblick auf die weitere Integration zu wagen (IV.).

\section{Die Aussagen des Bundesverfassungsgerichts zur Weiterentwicklung der Europäischen Union}

\section{Ausgangspunkt: Art. 38 Abs. 1 S. 1 GG}

Ausgehend von der prozessualen Grundlage des Verfahrens wird im Lissabon-Urteil zunächst das Zustimmungsgesetz zum Vertrag von Lissabon ${ }^{5}$ auf seine Vereinbarkeit mit dem Grundgesetz hin überprüft. Als Maßstab und Ausgang der Prüfung fungiert hierbei Art. 38 Abs. 1 S. 1 GG. ${ }^{6}$ Das Bundesverfassungsgericht betont erneut, ${ }^{7}$ dass die demokratische und periodische Wahl der Abgeordneten des Deutschen Bundestages, deren Ausübungsrecht in Art. 38 Abs. 1 S. 1 GG normiert wird, die Quelle der Staatsgewalt auf Bundesebene darstelle; in Zusammenhang mit Art. 20 Abs. 1 und 2 GG sieht es hierin ein Recht des Einzelnen begründet, eine Verletzung demokratischer Grundsätze durch Erhebung einer Individualverfassungsbeschwerde zu rügen. ${ }^{8}$ Weiter wird festgestellt, dass durch eine wesentliche Schmälerung der Rechte des Deutschen Bundestages unter Einhergehung eines Substanzverlustes demokratischer Gestaltungsmacht das „Prinzip der repräsentativen Volksherrschaft“ verletzt sein könne. ${ }^{9}$ Gleiches gilt, wenn der Deutsche Bundestag durch Abgabe von Kompetenzen auf die europäische Ebene nicht mehr in der Lage wäre, seine ihm als Kernaufgaben übertragenen Aufgaben wahrzunehmen. ${ }^{10}$ Darüber hinaus können auch vermeintliche Demokratiedefizite auf europäischer Ebene und letztlich auch Art. 146 GG - wenn auch nicht selbständig, so doch über Art. 38 Abs. 1 S. 1 GG - gerügt werden. ${ }^{11}$

4 Diese Parallelität bereits erkennend und aufzeigend: E. Röper, Der Souveränitäts- und Volksbegriff des Bundesverfassungsgerichts, in: DÖV 2010, S. 285 ff., 292; ebenso etwa : M. Selmayer, Endstation Lissabon? - Zehn Thesen zum „Niemals“-Urteil des Bundesverfassungsgerichts vom 30. Juni 2009, in: ZEuS 2009, S. 637 ff., 646; K. F. Gärditz/C. Hillgruber (Fn. 2), S. $875 \mathrm{f}$.

5 Gesetz vom 8. Oktober 2008 zum Vertrag von Lissabon vom 13. Dezember 2007, BGBl. 2008 Teil II Nr. 27 vom 14. Oktober 2008, S. 1038 ff.

6 BVerfGE 123, 267 ff., 340; so auch bereits im Maastricht-Urteil, BVerfGE 89, 155 ff., 182 ff.

7 So auch bereits in BVerfGE 89, $155 \mathrm{ff} ., 171$.

8 BVerfGE 123, $267 \mathrm{ff} ., 340$.

9 BVerfGE 123, $267 \mathrm{ff} ., 341$.

10 BVerfGE 123, $267 \mathrm{ff} ., 330$.

11 BVerfGE 123, $267 \mathrm{ff}$., $331 \mathrm{f}$. 


\section{Anspruch auf demokratische Teilhabe aus Art. 1 Abs. 1 GG}

Den Anspruch des Einzelnen auf Teilhabe im demokratischen Verfassungsstaat und damit auf Partizipation an der repräsentativen Volksherrschaft stützt das Urteil im Kern auf die Menschenwürde aus Art. 1 Abs. 1 GG als unveränderliches Wesensmerkmal des Grundgesetzes, wobei das Bundesverfassungsgericht einen diesbezüglichen subjektivrechtlichen Anspruch nicht aus Art. 1 Abs. 1 GG direkt, sondern „nur“ aus Art. 38 Abs. 1 GG ableitet. ${ }^{12}$ Das Bundesverfassungsgericht begründet seine Sichtweise damit, dass innerhalb der deutschen verfassungsmäßigen Ordnung letztlich alle den Bürger betreffende, bindende Entscheidungen auf einen ,frei gebildeten Mehrheitswillen des Volkes“ zurückzuführen sein müssten: Die verfassungsgemäße Ordnung baue auf der „Selbstbestimmung des Volkes“ und damit letztlich auf der Freiheit des Einzelnen auf, wie sie in Art. 1 Abs. 1 GG zum Ausdruck komme, und sei somit Ausdruck des in der Würde angelegten Eigenwertes des Menschen. ${ }^{13}$ Den Wahlen komme für die Teilhabe des Einzelnen im demokratischen Verfassungsstaat eine zentrale und tragende Rolle zu: Der Wahlakt projiziere die Mehrheitsmeinung in das Parlament, woraus sich letztlich Regierungen bilden lassen; diese können jedoch gleichzeitig durch selbigen Akt abgewählt werden. ${ }^{14}$ Diese Teilhabe des Einzelnen wirke ,,insbesondere im Status des Abgeordneten" fort, woraus die gleiche Teilhabe am Willensbildungsprozess im Deutschen Bundestag resultiere. ${ }^{15}$

\section{Garantie der souveränen Staatlichkeit Deutschlands}

Unter Zugrundelegung dieser Erwägungen als Grundpfeiler der eigenen Argumentation, prägt das Bundesverfassungsgericht das Schlagwort der „Garantie der souveränen Staatlichkeit" "16. Das Grundgesetz setze souveräne Staatlichkeit nicht nur voraus, sondern garantiere diese Staatlichkeit eben auch. ${ }^{17}$ Da das Demokratieprinzip, zudem das Recht auf Teilhabe elementar gehöre, einer Abwägung entzogen ist und die Inhalte der Art. 1 und 20 GG sich im geltenden Grundgesetz aufgrund der Ewigkeitsgarantie des Art. 79 Abs. 3 GG als unabänderbar darstellen, sei auch „dem verfassungsgebenden Gesetzgeber" ${ }^{18}$ dem pouvoir constitué, eine Änderung des Grundgesetzes in dieser Hinsicht verwehrt. ${ }^{19}$ Würde der Gesetzgeber diese festgelegte Grenze überschreiten, wäre dies ein unzulässiger „Übergriff in die verfassungsgebende Gewalt des Volkes“,

12 C. Enders, in: K. Stern/F. Becker, Grundrechte-Kommentar, 2010, Art. 1 GG, Rn. 45.

13 BVerfGE 123, 267 ff., 341; zu Recht kritisch gegenüber den Annahmen des Bundesverfassungsgerichts: C. Enders (Fn. 12), Rn. 27.

14 BVerfGE 123, $267 \mathrm{ff}$., $341 \mathrm{f}$.

15 BVerfGE 123, $267 \mathrm{ff} ., 342$.

16 BVerfGE 123, 267 ff., 343; zu dieser Feststellung und deren Bewertung siehe im zahlreichen Schrifttum etwa nur: M. Jestaedt (Fn. 2), S. 505 ff.

17 BVerfGE 123, 267 ff., 343.

18 Einer Beantwortung der Frage, ob dies auch für die ,,verfassungsgebende Gewalt“ selbst gelten würde, entzieht sich das Bundesverfassungsgericht jedoch und lässt diesen Punkt, in einem ansonsten durchaus breit angelegten Urteil offen; BVerfGE 123, 267 ff., 343.

19 BVerfGE 123, 267 ff., 343. 
des pouvoir constituant, der den Gesetzgeber zu einem solchen Schritt nicht ermächtigt hätte. ${ }^{20}$

\section{Offenheit für Integration}

Dieser „Garantie der souveränen Staatlichkeit“ stellt das Bundesverfassungsgericht jedoch seine Ausführungen zur Integrationsfreundlichkeit und -offenheit des Grundgesetzes gegenüber. Das Gericht erkennt an, dass der grundgesetzliche Integrationsauftrag sogar eine Integration in ein vom deutschen Grundgesetz verschiedenes System politischer Willensbildung ermöglicht, begrenzt diesen Integrationsauftrag jedoch durch die der Ewigkeitsklausel unterfallenden Grundgesetzbestandteile. ${ }^{21}$ Jenseits dieses grundgesetzlich geschützten Kernbestandes sei das Grundgesetz freilich auf Öffnung hin zur europäischen Integration ausgerichtet. ${ }^{22}$ An dieser Stelle bringt das Bundesverfassungsgericht nun den grundsätzlichen wie auch spezifizierten Grundrechtsauftrag zur europäischen Integration ins Spiel. Der Verweis auf die Präambel des Grundgesetzes, die als generelles Integrationsziel Deutschland als gleichberechtigtes Mitglied in einem vereinten Europa avisiert, und auf Art. 23 GG als Konkretisierung dieses recht opaken Auftrags der Präambel ${ }^{23}$ eröffnet anschließend die seither ausgiebig diskutierte ${ }^{24}$ Einbzw. Begrenzung des grundgesetzlichen Integrationsauftrages durch das Bundesverfassungsgericht. ${ }^{25}$

\section{Unmöglichkeit des „Bundesstaates“ nach dem Grundgesetz?}

Nach der Betonung, dass eine europäische Integration vom Grundgesetz nicht nur geduldet, sondern sogar eingefordert werde, ${ }^{26}$ verweist das Urteil zunächst darauf, dass mit dem Integrationsauftrag keine Festlegung bezüglich der Ausgestaltung der Europä-

20 BVerfGE 123, $267 \mathrm{ff} ., 344$.

21 BVerfGE 123, $267 \mathrm{ff} ., 344$.

22 BVerfGE 123, $267 \mathrm{ff} ., 344 \mathrm{f}$.

23 Vgl. hierzu etwa: C. Hillgruber, in: B. Schmidt-Bleibtreu/H. Hofmann/A. Hopfauf, GGKommentar, 12. Auflage 2011, Art. 23 GG, Rn. 7; C. D. Classen, in: H. von Mangoldt/F. Klein/C. Starck, GG-Kommentar, Band II, 6. Auflage 2010, Art. 23 GG, Rn. 1 ff.

24 Vgl. inter alia P.-C. Müller-Graff, Das Karlsruher Lissabon-Urteil: Bedingungen, Grenzen, Orakel und integrative Optionen, in: integration 4 (2009), S. 331 ff., 339 ff.; C. Schönberger, Lisbon in Karlsruhe: Maastricht's Epigones At Sea, in: German Law Journal 10 (2009), S. 1201 ff.; 1208 ff.; C. Ohler (Fn. 2), S. 172 ff.; W. Frenz, Nicht hinreichend demokratische EU? - Konsequenzen des Lissabon-Urteils des BVerfG vom 30.6.2009, in: EWS 2009, S. 441 ff., 443 f.; U. Everling, Europas Zukunft unter Kontrolle nationaler Verfassungsgerichte - Anmerkungen zum Urteil des Bundesverfassungsgerichts vom 30. Juni 2009 über den Vertrag von Lissabon, in: EuR 2010, S. 91 ff., 98 ff.; E. Denninger (Fn. 2), S. 971 ff.; E. Pache, Das Ende der europäischen Integration? - Das Urteil des Bundesverfassungsgerichts zum Vertrag von Lissabon, zur Zukunft Europas und der Demokratie, in: EuGRZ 2009, S. $285 \mathrm{ff} ., 290 \mathrm{ff}$.

25 BVerfGE 123, $267 \mathrm{ff} ., 345 \mathrm{ff}$.

26 BVerfGE 123, $267 \mathrm{ff} ., 346 \mathrm{f}$. 
ischen Union einhergeht. ${ }^{27}$ Dennoch sei es mit dem Grundgesetz jedenfalls nicht vereinbar, dass die deutschen Verfassungsorgane ,das Selbstbestimmungsrecht des Deutschen Volkes in Gestalt der völkerrechtlichen Souveränität Deutschlands auf[gäben]. "28 Daraus ergibt sich für das Bundesverfassungsgericht mithin ein Verbot des Beitritts Deutschlands zu einem ,europäischen Bundesstaat“ auf Grundlage des geltenden Grundgesetzes. ${ }^{29}$ Ein Beitritt zu einer derart verfassten Europäischen Union sei nur durch einen „unmittelbar erklärten Willen des Deutschen Volkes“ möglich; innerhalb des bestehenden Grundgesetzsystems sei ein solcher Schritt jedenfalls ausgeschlossen. ${ }^{30}$ Zwar verweise Art. 23 Abs. 1 S. 3 GG auf eine Anpassung des Grundgesetzes an Veränderungen innerhalb der Europäischen Union, allerdings fände auch eine solche Anpassung ihre Grenzen in der Ewigkeitsklausel des Art. 79 Abs. 3 GG und damit in der legaliter unabänderlichen Souveränität Deutschlands. ${ }^{31}$ Das Bundesverfassungsgericht sieht daher keine Möglichkeit für die Verwirklichung eines europäischen Bundesstaates auf dem Boden des geltenden Grundgesetzes durch den grundgesetzändernden Gesetzgeber. ${ }^{32}$ Denn es könne ,für die europäische Unionsgewalt kein eigenständiges Legitimationssubjekt geben, das sich unabgeleitet von fremdem Willen und damit aus eigenem Recht gleichsam auf höherer Ebene verfassen könnte. “33 Das Bundverfassungsgericht bleibt in den folgenden Ausführungen daher dem Modell des „Staatenverbundes ${ }^{\text {*34 }}$ treu. $^{35}$

\section{Weiterentwicklung}

Dem Lissabon-Urteil ist damit zunächst eine Negativeingrenzung zu entnehmen. Europäische Integration ist unter dem geltenden Grundgesetz nur höchstens bis zur Grenze des europäischen Bundesstaates unter Wahrung der vom Bundesverfassungsgericht weiter spezifizierten Kernbereiche staatlicher Gestaltung ${ }^{36}$ möglich. ${ }^{37}$ Wäre diese Grenze von Staatenverbundseite aus erreicht, dürfte eine weitergehende Integration nicht mehr durch den grundgesetzändernden Gesetzgeber vorgenommen werden. Das Kon-

27 BVerfGE 123, 267 ff., 347.

28 BVerfGE 123, $267 \mathrm{ff} ., 348$.

29 BVerfGE 123, 267 ff., 347 f., auch 332.

30 BVerfGE 123, $267 \mathrm{ff} ., 348$.

31 BVerfGE 123, 267 ff., $348 \mathrm{f}$.

32 Ausdrücklich zustimmend K. F. Gärditz/C. Hillgruber (Fn. 2), S. 875; inter alia kritisch und diesen Passagen des Urteils eine rechtliche Bindung absprechend E. Pache (Fn. 24), S. 298; kritisch auch etwa M. Niedobitek, The Lisbon Case of 30 June 2009 - A Comment from the European Law Perspective, in: German Law Journal 10 (2008), S. 1267 ff., 1269 f.; C. Schönberger (Fn. 2), S. $555 \mathrm{ff}$.

33 BVerfGE 123, $267 \mathrm{ff} ., 349$.

34 Der von P. Kirchhof (in: ders., Der Deutsche Staat im Prozeß der europäischen Integration, in: HStR VII, 1992, § 183, Rn. 38, 50 ff.) geprägte Begriff bleibt also weiterhin Richtschnur verfassungsrechtlicher Rechtsprechung in Deutschland.

35 BVerfGE 123, 267 ff., 348.

36 BVerfGE 123, $267 \mathrm{ff}$., $358 \mathrm{ff}$.

37 BVerfGE 123, 267 ff., 350; C. Tomuschat (Fn. 2), S. 273 deutet die Aussagen des Gerichts dahingehend, dass mit dem Vertrag von Lissabon nach den Vorgaben des Gerichts das Ende der grundrechtskonformen Integration erreicht sei. 
zept des „Staatenverbundes“ und damit die Konzeption der Mitgliedstaaten als Herren der Verträge und der abgeleiteten Ausübung von Hoheitsrechten prägen weiterhin und in verstärktem Maße die Rechtsprechung des Bundesverfassungsgerichts zur europäischen Integration.

\section{Unumgänglichkeit der Argumentation des Bundesverfassungsgerichts?}

Damit könnte man nun davon ausgehen, dass den Diskussionen um eine Weiterentwicklung und den immer wieder ins Feld geführten Forderungen nach einer weiteren „Politisierung“ der Europäischen Union durch das Lissabon-Urteil ein jähes Ende bereitet wurde. Dass eine vom Bundesverfassungsgericht dargelegte Argumentation und eindeutige Festlegung hinsichtlich der Begrenzung der Integration in die Europäische Union zwar aus dem etatischen Denken ${ }^{38}$ des Gerichts heraus nachvollziehbar, jedoch nicht zwingend ist, wird folgend unter kritischer Würdigung der Darlegungen des Bundesverfassungsgerichts thematisiert werden.

\section{Art. 38 Abs. 1 S. 1 GG zu weit verstanden}

a) Wie bereits in seinem Maastricht-Urteil ${ }^{39}$ hat das Bundesverfassungsgericht im Lissabon-Urteil Art. 38 Abs. 1 S. 1 GG über seinen direkten Wortlaut hinaus interpretiert und dargelegt, dass neben der bloßen Teilnahme des Bürgers an Wahlen zum Deutschen Bundestag auch das Vorliegen von Gestaltungsmacht für das zu wählende Parlament durch Art. 38 Abs. 1 S. 1 GG geschützt sei; bei „Entleerung“ dieser Gestaltungsmacht werde das Demokratieprinzip, wie es in Art. 20 Abs. 1 und 2 GG niedergelegt und durch Art. 79 Abs. 3 GG garantiert sei, verletzt. ${ }^{40}$ Diese Interpretation des Art. 38 Abs. 1 S. 1 GG ergibt sich unterdessen nicht aus dessen Wortlaut, der lediglich die Wahl zum Deutschen Bundestag bzw. dessen Abgeordneten als solche vorschreibt und die Wahlrechtsgrundsätze für eine solche Wahl festlegt. Es ist daher vom Wortlaut her nicht ersichtlich, warum diese Norm eine inhaltliche Erweiterung erfahren soll. ${ }^{41}$ Zwar kommt der Wahl zum Deutschen Bundestag als Ausdruck des Volkswillens innerhalb der demokratischen Struktur des Grundgesetzes eine grundlegende Bedeutung zu. Eine Implementation dieses Gestaltungswillens in einer Norm des Grundgesetzes, die sich in eine Gruppe von Normen mit rein statuserläuternden und technischen Inhalten einreiht, erscheint jedoch zumindest nicht zwingend und im Grunde weit hergeholt. Es scheint vielmehr, dass dieser „Kunstgriff“42 nötig ist, um in der Sache entscheiden zu

38 Zur Kritik hierzu siehe inter alia P. Häberle (Fn. 2), S. 329 ff.; R. Bieber (Fn. 2), S. 396 ff.; M. Ruffert (Fn. 2), S. 1198 f., 1207; D. Halberstam/C. Möllers (Fn. 2), S. 1249 f.

39 Dort BVerfGE 89, 155 ff., 171 f., 182 ff., 187.

40 BVerfGE 89, 155 ff., 172; BVerfGE 123, 267 ff., 330, 340 f.; ebenso kurz: D. Grimm (Fn. 2), S. 481.

41 M. Jestaedt (Fn. 2), S. 503 f.; diese Frage wurde bereits zum Maastricht-Urteil zu Recht erörtert. Zur berechtigten damaligen Kritik hieran siehe neben vielen C. Tomuschat, Die Europäische Union unter Aufsicht des Bundesverfassungsgerichts, in: EuGRZ 1993, S. 489 ff., 489, 491.

42 J. P. Terhechte (Fn. 2), S. 726. 
können. Es dürften sich daher, wie bereits vielfach andernorts bemerkt, wohl rein verfahrenstaktische Gründe hinter dieser Aufladung des Art. 38 Abs. 1 S. 1 GG verbergen. ${ }^{43}$

b) Das Bundesverfassungsgericht nimmt in seinem Lissabon-Urteil nicht nur seine Interpretation des Art. 38 Abs. 1 S. 1 GG aus dem Maastricht-Urteil auf, sondern erweitert den Gehalt zudem. ${ }^{44}$ Zur Gehaltserweiterung des Art. 38 Abs. 1 S. 1 GG als individuelles Recht gegen eine „Entleerung“ der Entscheidungsgewalt des Deutschen Bundestages tritt die Möglichkeit, die Europäische Union auf ihren Demokratiegehalt hin zu überprüfen, ${ }^{45}$ die Verletzung des Sozialstaatsprinzips und weiterer Staatsstrukturprinzipien $^{46}$ (im Rahmen des Demokratieprinzips und im Mindestgehalt der Art. 23 Abs. 1 S. 3 i.V.m. Art. 79 Abs. 3 GG) $)^{47}$ zu rügen $^{48}$ und sogar Art. 146 GG i.V.m. Art. 38 Abs. 1 S. $1 \mathrm{GG}^{49}$ individuell rechtlich überprüfen zu lassen. Mit seiner „Verkettung“ von Demokratieprinzip und Wahlrecht und der darüber hinausgehenden Verknüpfung mit dem Begriff der Souveränität ${ }^{50}$ hat das Bundesverfassungsgericht zwar die Möglichkeit begründet, in einem weiten, bisher unbekannten Ausmaß Verfassungsbeschwerden erheben zu können. ${ }^{51}$ Dies wirft indes die Frage auf, ob die Möglichkeit, eine „Entleerung“ der Rechte des Deutschen Bundestages zu rügen, nicht zunächst voraussetzt, dass der Wähler einen Substanzverlust - da an einem klaren Maßstab messbar - auch erkennen kann und deshalb sein vermeintlich verletztes, auf dem Wahlrecht fußendes Recht geltend machen will. Und selbst wenn ein solcher Erkenntnisprozess vorläge, was anhand der Komplexität der Entscheidungsstrukturen zumindest fraglich ist, wann wäre ein Maß erreicht, dass nicht mehr hinnehmbar ist? ${ }^{52}$ Das Gericht rekurriert hier auf die Umschreibung des ,nicht mehr ausreichenden“ 53 Gestaltungsspielraumes für den deutschen Gesetzgeber. Wahrlich weiter hilft sie jedenfalls nicht. ${ }^{54}$

c) Wenn das Wahlrecht ein elementarer Bestandteil des Demokratieprinzips ist (und dieses über Art. 38 Abs. 1 S. 1 GG zur Abwehr einer „Entleerung“ der Gestaltungsmacht des Deutschen Bundestages zugleich gerügt werden kann) und zweifelsohne das Mehrheitsprinzip und die Möglichkeit der Abwahl von derzeitigen Mehrheiten zugunsten der Opposition prägende Facetten des Demokratieprinzips bilden, dann ist zudem fraglich,

43 So inter alia M. Jestaedt (Fn. 2), S. 504; J. P. Terhechte (Fn. 2), S. 726.

44 C. Schönberger (Fn. 2), S. 540, 541 ff.; M. Wendel (Fn. 2), S. 108 ff.; P.-C. Müller-Graff (Fn. 24), S. 336 ff.; M. Kottmann/C. Wohlfahrt (Fn.2), S. 464 f.; C. Tomuschat (Fn. 2), S. $264 \mathrm{ff}$.

45 BVerfGE 123, 267 ff., 331.

46 D. Thym (Fn. 2), S. 560.

47 BVerfGE 123, $267 \mathrm{ff} ., 332 \mathrm{f}$.

48 BVerfGE 123, $267 \mathrm{ff} ., 332 \mathrm{f}$.

49 BVerfGE 123, 367 ff., 332; kritisch zumindest für diese Ausweitung: M. Ruffert (Fn. 2), S. 1206.

50 D. Grimm (Fn. 2), S. 480 f.; P.-C. Müller-Graff(Fn. 24), S. 339.

51 Kritisch gegenüber dieser „Einladung“ zur Erhebung einer Verfassungsbeschwerde etwa $E$. Pache (Fn. 24), S. $287 \mathrm{ff}$.

52 P.-C. Müller-Graff (Fn. 24), S. 337.

53 BVerfGE 123, $267 \mathrm{ff} ., 358$.

54 Vgl. E. Denninger (Fn. 2), S. 972; P.-C. Müller-Graff(Fn. 24), S. 337. 
ob die vom Bundesverfassungsgericht zu Art. 38 Abs. 1 S.1 GG in diesem Zusammenhang dargelegten Grundsätze überhaupt eine Änderung in der derzeitigen politischen Realität Deutschlands bewirken. Denn gegenwärtig gibt es wohl keine mehrheitsfähige Koalitionsoption, die einer Weiterentwicklung der Europäischen Union über das heute erreichte Maß hinaus im Grundsatz entgegenstehen würde. Die Unterschiede in der nationalen Europapolitik sind schließlich eher fachspezifischer, denn grundsätzlicher Natur. ${ }^{55}$

d) Ferner ist zu beachten, dass gemäß Art. 23 Abs. 1 S. 1 GG als Ausprägung des Integrationsauftrages der Präambel des Grundgesetzes eine Integration nur in eine Europäische Union erfolgen kann, die demokratischen Grundsätzen verpflichtet ist. Das Bundesverfassungsgericht spricht aber selbst davon, dass die demokratischen Grundsätze institutionsbedingt auf europäischer Ebene anders zu beurteilen sind als in Deutschland. ${ }^{56}$ Wenn dem aber richtigerweise so ist, kann dann über Art. 38 Abs. 1 S. 1 GG wirklich eine Kontrolle nach dem Grundgesetz erfolgen ${ }^{57}$ Und wozu bedarf es überhaupt dieses Rückgriffs, wenn doch Art. 23 Abs. 1 S. 1 GG bereits ein demokratisches Mindestmaß garantiert, da eine Kompetenz nur insoweit abgegeben werden kann, als die Europäische Union weitestgehend demokratischen Grundsätzen entspricht? ${ }^{58} \mathrm{Be}$ darf es dann einer erneuten Prüfung der demokratischen Legitimität der Europäischen Union über Art. 38 Abs. 1 S. 1 GG als subjektives Recht? Oder anders gefragt: Wenn die Europäische Union demokratischen Grundsätzen soweit genügt, dass man Kompetenzen gemäß Art. 23 Abs. 1 S. 1 GG auf sie übertragen kann, ${ }^{59}$ ist dann noch Raum für rügbare Defizite über Art. 38 Abs. 1 S. 1 GG? ${ }^{60}$

Insgesamt wird die Ausweitung der auf Art. 38 Abs. S. 1 GG zu stützenden rügefähigen Rechte vom Bundesverfassungsgericht zwar postuliert, aber leider keiner näheren Begründung unterzogen. ${ }^{61}$

55 Hierauf ebenso verweisend: M. Nettesheim, Die Karlsruher Verkündung - Das BVerfG in staatsrechtlicher Endzeitstimmung, in: EuR 2010, S. 101 ff., 117; C. Schönberger (Fn. 24), S. 1217.

56 BVerfGE 123, 267 ff., 347.

57 So richtigerweise bereits E. Pache (Fn. 24), S. 291.

58 Aufgrund der erkannten Demokratiedefizite nimmt das Bundesverfassungsgericht eine Legitimation über die Nationalstaaten an; vgl. BVerfGE 123, 267 ff., 364.

59 Beachte: „Das Grundgesetz sagt „Ja“ zum Vertrag von Lissabon.“ (A. Voßkuhle), so mit berechtigter Kritik an diesem Ausspruch M. Jestaedt (Fn. 2), S. 499, 510.

60 So die Überlegungen im Zusammenhang mit den Aussagen des Gerichts zu Art. 79 Abs. 3 GG bei C. Tomuschat (Fn. 2), S. 278 f.; darüber hinaus die Überlegungen bei M. Jestaedt (Fn. 2), S. 510 f.; in diese Richtung wohl auch J. Sack, Der „Staatenverbund“ - Das Europa der Vaterländer des Bundesverfassungsgerichts, in: ZEuS 2009, S. 623 ff., 628; C. Calliess, Das Ringen des Zweiten Senats mit der Europäischen Union: Über das Ziel hinaus geschossen..., in: ZEuS 2009, S. 559 ff., 573.

61 Vgl. inter alia: D. Thym (Fn. 2), S. 560; zu Recht äußerst kritisch und die Rückführung auf die Zulässigkeitsvoraussetzung der unmittelbaren Selbstbetroffenheit bei Grundrechtsverstößen anmahnend: C. Tomuschat (Fn. 2), S. 265 ff. 


\section{Die Menschenwürde des Art. 1 Abs. 1 GG}

Ebenso eine Mehrfachverschränkung stellt der Rekurs des Bundesverfassungsgerichts auf die Menschenwürde des Art. 1 Abs. 1 GG in Zusammenhang mit der Teilhabe des einzelnen Bürgers an der öffentlichen Gewalt dar. ${ }^{62}$ Zwar ist dem Bundesverfassungsgericht insoweit zuzustimmen, als Ausgangspunkt des Demokratieprinzips letztlich stets die Freiheit und die freie Willensbildung des Menschen in seiner durch Art. 1 Abs. 1 GG geschützten Sphäre sein muss. ${ }^{63}$ Allerdings ist dieses Prinzip der freien Willensbetätigung des Einzelnen in einer bestehenden Gesellschaftsstruktur eben bereits durch Art. 20 Abs. 1 und 2 GG geschützt. ${ }^{64}$ Die Stoßrichtung der Grundrechte geht zwar von einer Beteiligung in einer demokratisch verfassten Gemeinschaft aus, stellt aber auf das Individuum selbst und dessen Abgrenzung nach außen ab; es bedurfte daher eines solchen Rekurses auf Art. 1 Abs. 1 GG zum Schutz der Teilhabe des Bürgers an der öffentlichen Gewalt nicht. ${ }^{65}$

Man darf sich also zu Recht fragen, was diese Mehrfachabsicherung am Ende bezwecken sollte und vor allem, inwieweit diese Erwägungen dann überhaupt Entscheidungsrelevanz aufweisen. ${ }^{66}$ Dies gilt umso mehr, als das Demokratieprinzip selbst bereits von der Ewigkeitsklausel des Art. 79 Abs. 3 GG erfasst ist und mit Art. 1 Abs. 1 GG somit auch in diesem Zusammenhang keine Änderung des Schutzes vor Beeinträchtigung einhergeht. Auch ohne diesen Rekurs ist das Demokratieprinzip „unantastbar““ 67

\section{Widerspruch zwischen Integrationsoffenheit und Garantie der Staatlichkeit einseitig entschieden $^{68}$}

a) Als wären nicht schon genug sehr weit gefasste Interpretationen im Urteil vorhanden, setzt das Bundesverfassungsgericht schließlich zu einer weiteren interpretativen Ausdehnung an. ${ }^{69}$ Zwar wird erstmals nicht nur die Möglichkeit der Integration in die Europäische Union betont, sondern sogar von einer Integrationspflicht gesprochen. ${ }^{70}$ Den-

62 C. Enders (Fn. 12), Rn. 27 f.; BVerfGE 123, 267 ff., 341; diesen Rekurs ausdrücklich begrüßend: P. Häberle (Fn. 2), S. 322; ebenso: F. Schorkopf, Die Europäische Union im Lot Karlsruhes Rechtsspruch zum Vertrag von Lissabon, in: EuZW 2009, S. 718 ff., 719; eine weitere Ausweitung der Verfassungskontrolle über Art. 1 GG vorhersagend: F. Schorkopf (Fn. 2), S. 1233.

63 So etwa K. F. Gärditz/C. Hillgruber (Fn. 2), S. 872 f.

64 C. Enders (Fn. 12), Rn. 27 f; C. Schönberger (Fn. 2), S. 554.

65 C. Enders (Fn. 12), Rn. $27 \mathrm{f}$.

66 Kritisch zum Rekurs auf Art. 1 Abs. 1 GG inter alia: C. Schönberger (Fn. 2), S. 541 f., 554; ders. (Fn. 24), S. 1208, mit Bezug auf Art. 79 Abs. 3 GG; zur Kritik an den vielen Äußerungen außerhalb des Prüfungsumfangs des Bundesverfassungsgerichts siehe inter alia: $R$. Wahl (Fn. 2), S. 613; D. Grimm (Fn. 2), S. 492; P. Häberle ([Fn. 2], S. 321) spricht gar von einer „habilitationsgleiche[n] Leistung“; C. Calliess (Fn. 60), S. 565.

67 BVerfGE 123, 267 ff., 341, 343; C. Schönberger (Fn. 2), S. 554.

68 So im Ergebnis auch C. Tomuschat (Fn. 2), S.271; nach der Auswirkung der Europarechtsfreundlichkeit fragend auch M. Ruffert (Fn. 2), S. 1207.

69 Zur Interpretation des Art. 79 Abs. 3 GG vgl. inter alia M. Jestaedt (Fn. 2), S. 505 ff.; C. Tomuschat (Fn. 2), S. 278 f.; D. Halberstam/C. Möllers (Fn. 2), S. 1253 f.

70 BVerfGE 123, $267 \mathrm{ff}$., $346 \mathrm{f}$. 
noch wird dieser aus der Präambel und Art. 23 Abs. 1 GG fließenden Erkenntnis kaum mehr Wirkung eingeräumt als einem grundsätzlichen Programmsatz, der aber ohne weitere Auswirkungen bleiben dürfte. ${ }^{71}$ Denn wenn das Bundesverfassungsgericht das Zustimmungsgesetz zum Vertrag von Lissabon unter den eigens aufgestellten Bedingungen $^{72}$ als ,,noch“73 mit dem Grundgesetz vereinbar ansieht (wenn auch Weiterentwicklungen bis zur „Entleerung“" staatlicher Gestaltungsmacht noch in sehr engen Grenzen weiter möglich erscheinen) ${ }^{74} 75$ die Entwicklung der Europäischen Union hin zu einem ,staatenähnlichen“ Gebilde aber als mit dem geltenden Grundgesetz unvereinbar erklärt, ${ }^{76}$ wird die neu erteilte Pflicht zur europäischen Integration wohl gleichsam als eine Pflicht zur Durchführung eines untauglichen Versuchs anzusehen sein.

b) Das Bundesverfassungsgericht betont an mehreren Stellen seines Urteils die Integrationsoffenheit und -pflicht des deutschen Grundgesetzes ${ }^{77}$ und stellt zudem fest, dass weder Präambel noch Art. 23 Abs. 1 GG eine finale Festlegung bezüglich der Verfasstheit der Europäischen Union vorgeben. Auch sieht es Abweichungen dieser Verfasstheit von Vorgaben, wie sie das Grundgesetz für Deutschland trifft (unter Verweis auf Art. 28 Abs. 1 S. 1 GG), aufgrund der speziellen Eigenart einer supranationalen Gemeinschaft für möglich an. ${ }^{78}$ Sogar das Leitbild und die Implementation einer ,politischen Union" werden vom Bundesverfassungsgericht als verfassungsrechtlich zulässig erachtet, ${ }^{79}$ und der Überwindung der unfriedvollen Vergangenheit wird der Status eines überragenden politischen Ziels der Bundesrepublik eingeräumt. ${ }^{80}$ Trotz dieser Affirmation folgt hierauf eine Eingrenzung unter Bezugnahme auf die Ewigkeitsgarantie des Art. 79 Abs. 3 GG: Eine Integration sei begrenzt durch die staatliche Souveränität. ${ }^{81}$ Diese Eingrenzung erscheint jedoch nicht zwingend geboten. Die Ausweitung des Art. 79 Abs. 3 GG unter Berufung auf das Demokratieprinzip und die staatliche Souveränität wurde richtigerweise als mit seiner Entstehungsgeschichte nicht begründbar angesehen. ${ }^{82}$ Art. 79 Abs. 3 GG ist eben nicht darauf angelegt, den Verfassungsauftrag

71 So wohl auch M. Ruffert (Fn. 2), S. 1207.

72 BVerfGE 123, 267 ff., 369: ,nach Maßgabe der Gründe“; zu der hierzu geführten Diskussion eines völkerrechtlichen Vorbehalts bzgl. der Interpretationseinschränkungen durch das Bundesverfassungsgerichts bei Ratifizierung des Lissabon-Vertrages, siehe einen solchen fordernd: K. F. Gärditz/C. Hillgruber (Fn. 2), S. 877 f., a.A. C. Calliess (Fn. 60), S. 577 ff.

73 BVerfGE 123, $267 \mathrm{ff}$., $369 \mathrm{f}$.

74 Zur vagen Formulierung der als besonders sensibel aufgeführten Bereiche der staatlichen Gestaltungsmacht: D. Grimm (Fn. 2), S. $490 \mathrm{f}$.

75 So die Deutung der Passagen in BVerfGE 123, 267 ff., 369 f.; so auch: C. Tomuschat (Fn. 2), S. 273.

76 BVerfGE 123, 267 ff., 347 f., 349, 364 f., $368 \mathrm{f}$.

77 Vgl. etwa BVerfGE, 123, 267 ff., 346 f., 354, 401 bezogen auf eine „Europarechtsfreundlichkeit".

78 BVerfGE 123, 267 ff., 347.

79 BVerfGE 123, $267 \mathrm{ff} ., 357$.

80 BVerfGE 123, 267 ff., 346.

81 Garantie der souveränen Staatlichkeit; BVerfGE 123, 267 ff., 343, 347 f.; so auch C. Hillgruber, Der Nationalstaat in der überstaatlichen Verflechtung, in: HStR II, 2004, §32, Rn. 41, $107 \mathrm{f}$.

82 Inter alia: C. Tomuschat (Fn. 2), S. 278 f.; M. Jestaedt (Fn. 2), S. 506 f.; C. Schönberger (Fn. 2), S. 553 ff.; F. C. Mayer, Rashomon in Karlsruhe, in: NJW 2010, S. 714 ff., 716. 
der Präambel oder des Art. 23 Abs. 1 GG zu minimieren, sondern fungiert gleichsam als Abwehrrecht gegen einen Umsturz. ${ }^{83}$ Die Integration in eine Europäische Union, die demokratischen, rechtsstaatlichen, sozialen und föderativen Grundsätzen sowie dem Subsidiaritätsgedanken und einem dem Grundgesetz vergleichbaren Grundrechtsschutz verpflichtet ist (Art. 23 Abs. 1 S. 1 GG), kann mit dieser Ausnahme- und Extremsituation daher wohl kaum verglichen werden. ${ }^{84}$ Es erscheint daher weder notwendig noch erstrebenswert, ${ }^{85}$ Art. 79 Abs. 3 GG derart weit zu erstrecken, zumal, wie bereits oben dargelegt, eine Integration nur in eine demokratisch strukturierte Europäische Union überhaupt erfolgen kann. ${ }^{86}$ Der Einschätzung des Bundesverfassungsgerichts, dass derzeit auf europäischer Ebene ein solcher demokratischer Zustand für eine weitergehende Übertragung der Hoheitsrechte jenseits des bisherigen Integrationsstandes noch nicht erreicht ist, ${ }^{87}$ mag man zustimmen. Die Annahme, dass dieser aber nicht erreichbar sei, ${ }^{88}$ ist, sofern man die Annahmen des Bundesverfassungsgerichts zu Art. 79 Abs. 3 GG in Bezug auf die Endlichkeit der Integration nicht teilt, nicht stichhaltig dargelegt worden und übersieht die stetigen Bemühungen im Integrationsprozess um mehr demokratische Legitimation auf europäischer Ebene. ${ }^{89}$ Das Bundesverfassungsgericht belässt es bei seiner Feststellung, dass nach seiner dargelegten Argumentation ein Bundesstaat Europa verfassungsrechtlich derzeit ausgeschlossen sei, ${ }^{90}$ ohne ein Wort dazu zu verlieren, inwieweit weitere Entwicklungen und Änderungen des Primärrechts in dieser Hinsicht Einfluss nehmen könnten. Wenn das Bundesverfassungsgericht schon zu einer nicht gestellten Frage ${ }^{91}$ Stellung nimmt, wären hierzu auch weitere Ausführungen wünschenswert gewesen. ${ }^{92}$ Da das Bundesverfassungsgericht sich diesbezüglich jedoch verschließt, bleibt daraus nur abzuleiten, dass - gleich welche Entwicklungen auch immer die Zukunft bringen mag - eine Integration hin zum Bundesstaat unter dem geltenden Grundgesetz wegen der Garantie der Staatlichkeit nicht möglich ist. Dies führt letztlich zu dem in der Literatur oft beschriebenen Dilemma, dass die Europäische Union als demokratisch defizitär angesehen wird, gleichzeitig durch eine Weiterentwicklung in Richtung Demokratisierung einer Staatsgleichheit (und damit einem zumindest bundesstaatsähnlichen Gebilde) näher rückt, was jedoch gleichzeitig verfassungsrechtlich unzulässig ist. ${ }^{93}$ Richtigerweise sind jedoch nur die Staatsstrukturprinzipien, nicht zugleich die Staatlichkeit an sich, durch Art. 79 Abs. 3 GG

83 M. Jestaedt (Fn. 2), S. 507; grundsätzlich offener: D. Grimm (Fn. 2), S. 489.

84 D. Halberstam/C. Möllers (Fn. 2), S. 1254.

85 C. Tomuschat (Fn. 2), S. 279, weist zu Recht auf die Gefahr einer „Abnutzung“ von Art. 79 Abs. 3 GG hin.

86 C. Tomuschat (Fn. 2), S. 278.

87 BVerfGE 123, $267 \mathrm{ff} ., 370 \mathrm{ff}$.

88 Vgl. D. Grimm (Fn. 2), S. 490.

89 Siehe etwa die berechtigte Kritik bei D. Halberstam/C. Möllers (Fn. 2), S. 1247; P. Häberle (Fn. 2), S. 333.

90 Kritisch bezüglich einer fehlenden Begründung zur Ausweitung des Art. 79 Abs. 3 GG insgesamt: D. Thym (Fn. 2), S. 560.

91 Vgl. Fn. 32 und 66.

92 So auch D. Grimm (Fn. 2), S. 490.

93 Vgl. zu diesem mehrfach festgestellten Dilemma inter alia D. Grimm (Fn. 2), S. 489; D. Thym (Fn. 2), S. 576 ff.; D. Halberstam/C. Möllers (Fn. 2), S. 1251. 
geschützt. ${ }^{94}$ Ist zudem aus dem Integrationsauftrag des Art. 23 Abs. 1 S. 1 GG durch die Integrationsvoraussetzung ,demokratisch[...]" nicht gleichzeitig der Auftrag erteilt, an einer möglichst stetigen und fortschreitenden Demokratisierung der Europäischen Union mitzuwirken? Und ist demgemäß nicht auch ein Auftrag an den pouvoir constitué, die Europäische Union - wenn man wie das Bundesverfassungsgericht in staatlichen Denkstrukturen verhaftet bleiben will - zu einem Gebilde zu formen, das den möglichst höchsten Demokratieanforderungen entspricht? Dieser Auftrag wird vom Bundesverfassungsgericht in dieser Richtung nicht gesehen. Es geht eher davon aus, dass für Deutschland und das geltende Grundgesetz in einer höheren Demokratisierung eine Gefahr besteht, nämlich die Gefahr einer „Entstaatlichung“. Dabei übersieht das Gericht, wie zu Recht kritisiert wurde, ${ }^{95}$ dass ein Bundesstaat nicht einem Zentralstaat gleich zu stellen sein muss. ${ }^{96}$ Ein europäischer Bundesstaat, wenn man die Garantie der Staatlichkeit in derzeitigem Status nach dem Grundgesetz überhaupt als gegeben ansieht, muss nicht zwangsläufig in der Aufgabe der eigenen Staatlichkeit für die Gliedstaaten münden; vielmehr können auch die Gliedstaaten ihre Staatlichkeit durchaus behalten, wie das Beispiel der Bundesrepublik Deutschland deutlich zeigt. ${ }^{97}$

\section{Ausschluss des Bundesstaates}

Sieht man die Überdehnung des Art. 79 Abs. 3 GG in Bezug auf die Garantie der Staatlichkeit und die diesbezügliche Bindung des Art. 23 Abs. 1 S. 3 GG als nicht geboten und damit unzulässig an, so bleiben Art. 23 Abs. 1 GG als Staatszielbestimmung ${ }^{98}$ sowie die Präambel des Grundgesetzes als Integrationsprogramm. ${ }^{99}$ Beide schließen eine Integration in einen ,europäischen Bundesstaat“, wie er im Übrigen für die Entscheidung des Bundesverfassungsgerichts nicht zur Debatte stand, ${ }^{100}$ nicht aus, sondern stellen lediglich Anforderungen an die Ausgestaltung eines solchen „Bundesstaates“. ${ }^{101}$ An anderer Stelle wurde zudem darauf verwiesen, dass Art. 23 Abs. 1 GG in heutiger Form

94 C. Möllers, Staat als Argument, 2. Auflage 2011, S. 382 ff.; B.-O. Bryde, in: I. v. Münch/P. Kunig, GG-Kommentar, Band 3, 4./5. Auflage 2003, Art. 23 GG, Rn. 49 a; differenzierend, aber im Ergebnis wie hier: I. Pernice, in: H. Dreier (Hrsg.), GG-Kommentar, Band II, 2. Auflage 2006, Art. 23 GG, Rn. 36.

95 C. Schönberger (Fn. 2), S. 555 ff.

96 C. Schönberger (Fn. 24), S. 1215.

97 I. Pernice (Fn. 94), Rn. 36; C. Schönberger (Fn. 24), S. 1214 ff.; ders. (Fn. 2), S. 556; M. Selmayer (Fn. 4), S. 646; a.A. hinsichtlich der Qualität der Staatlichkeit D. Murswiek, Maastricht und der Pouvoir Constituant - Zur Bedeutung der verfassungsgebenden Gewalt im Prozeß der europäischen Integration, in: Der Staat 32 (1993), S. $161 \mathrm{ff.,} 164$ f.

98 C. Hillgruber (Fn. 23), Rn. 7.

99 M. Selmayer [(Fn. 4), S. 645] sieht sogar eine Verpflichtung der deutschen Staatsorgane, auf einen europäischen Bundesstaat hinzuwirken.

100 Wie inter alia E. Pache ([Fn. 24], S. 289) zu Recht anmerkt.

101 I. Pernice (Fn. 94), Rn. 36 f, 48 f.; im Ergebnis ebenso: M. Selmayer (Fn. 4), S. 646; J. Sack (Fn. 60), S. 625 ff.; wohl auch: W. Frenz (Fn. 24), S. 443, 445; F. C. Mayer (Fn. 82), S. 715 . 
erst in den neunziger Jahren in das Grundgesetz Einzug hielt und man damals bei den Erwägungen keine Begrenzung der europäischen Integration vorgenommen hat. ${ }^{102}$

Dies vorausgesetzt könnte das Demokratieprinzip nach entsprechender primärrechtlicher Entwicklung eines Tages auch vollständig auf europäischer Ebene verkörpert sein, ${ }^{103}$ wodurch eine Verletzung der durch Art. 79 Abs. 3 GG geschützten Staatsprinzipien vermieden werden könnte. Zwingend ist der Ausschluss des Bundesstaates nach geltendem Grundgesetz also wohl nicht. ${ }^{104}$

Dennoch hat das Bundesverfassungsgericht anders judiziert und damit erneut eine weitere interessante Frage aufgeworfen, die bereits lange in der Rechtswissenschaft diskutiert wird und auch verfassungsgeschichtliche Vorläufer aufweist. Vorausgesetzt man will politisch eine Entwicklung hin zu einem Bundesstaat Europa: Wie müsste die vom Bundesverfassungsgericht als zwingend angesehene „Abwahl des Grundgesetzes“ dann erfolgen? Das Gericht hält sich in dieser Frage eher bedeckt. Zwar verweist es an einigen Stellen in seinem Urteil auf Art. 146 GG. Einen wirklichen Weg weist es jedoch nicht auf. ${ }^{105}$

Nachdem bereits im Zuge der Deutschen Wiedervereinigung eine vergleichbare, wenn auch auf die Vereinigung zweier deutscher Staaten und damit nicht auf eine supranationale Bundesstaatsbildung ausgerichtete, Diskussion geführt wurde, soll im Folgenden die Art und Weise einer Neuverfassungsgebung diskutiert werden. ${ }^{106}$

\section{Wege zu einer neuen Verfassungsgebung}

\section{Aussagen des Bundesverfassungsgerichts}

Im Zuge der Feststellung der Inkompatibilität von deutschem Grundgesetz und „europäischem Bundesstaat" verweist das Bundesverfassungsgericht in seinem Urteil an einigen - vergleichsweise wenigen - Stellen auf Art. 146 GG.${ }^{107}$ Dabei stellt es fest, dass Art. 146 GG das vorverfassungsrechtliche Recht der Verfassungsgebung durch den wahlberechtigten Bürger bestätigt. Zudem stelle Art. 146 GG (neben den Anforderungen des Art. 23 Abs. 1 GG) die ,äußerste Grenze der Mitwirkung der Bundesrepublik

102 M. Jestaedt (Fn. 2), S. 507 f.; M. Selmayer (Fn. 4), S. 646; vgl. hierzu auch die Aussage in BT-Drs. 12/3338, S. 7 lit. d), dass es offen bleiben könne, ob für den Eintritt in einen europäischen Bundesstaat eine Verfassungsänderung ausreiche; zudem erfolgte ein Hinweis auf Meinungsverschiedenheiten der Sachverständigen in diesem Punkt in der gemeinsamen Verfassungskommission.

103 Vom Verfassungsgericht in dieser Hinsicht daher weitere Ausführungen einfordernd: $D$. Grimm (Fn. 2), S. 490; ders. (Fn. 2), S. 489, der ebenfalls eine Implementation der ,identitätsbegründenden Prinzipien [...], [...] auf höherer Ebene" nicht ausschließt; in diese Richtung auch M. Niedobitek (Fn. 32), S. $1269 \mathrm{f}$.

104 So wohl auch, da nur kurz erwähnt: P. Häberle (Fn. 2), S. 335 f.; einen europäischen Bundesstaat als möglich betrachtend, solange nicht die Staatlichkeit Deutschlands selbst in Frage gestellt werden soll: I. Pernice, (Fn. 94), Rn. 36 ff.; a.A. C. D. Classen (Fn. 23), Rn. 3, 6,19 , weil nur einzelne Hoheitsrechte übertragen werden dürften.

105 D. Thym (Fn. 2), S. 565.

106 Vgl. Referenzen in Fn. 4.

107 BVerfGE 123, 267 ff., 332, indirekt 343, 349. 
Deutschland an der europäischen Integration"108 dar. Im Zusammenhang mit der Unabwägbarkeit des Demokratieprinzips und der Garantie der souveränen Staatlichkeit Deutschlands dekretiert das Bundesverfassungsgericht, dass jedenfalls dem verfassungsgebenden Gesetzgeber eine Aufgabe der souveränen Staatlichkeit nicht erlaubt sei. Wie ebenfalls bereits erwähnt, lässt das Bundesverfassungsgericht explizit offen, ob dies auch für die ,verfassungsgebende Gewalt“ (pouvoir constituant) gelten kann und verweist auf einschlägige Literatur zu Art. 146 GG. ${ }^{109}$ Schließlich stellt das Bundesverfassungsgericht fest, dass der Integrationsauftrag des Art. 23 Abs. 1 GG in Verbindung mit der Präambel, Art. 20, Art. 79 Abs. 3 und Art. 146 GG nicht zu einem eigenständigen Legitimationssubjekt für die europäische Unionsgewalt führen könne, welches, unabgeleitet von fremdem Willen und damit aus eigenem Recht gleichsam auf höherer Ebene verfass[t] [werden] könne." ${ }^{110}$ Das heißt, dass der Eintritt in einen „europäischen Bundesstaat“ aus Sicht des Bundesverfassungsgerichts nicht mit dem geltenden Grundgesetz vereinbar ist; ein derartiger Integrationsschritt wäre ,allein dem unmittelbar erklärten Willen des Deutschen Volkes vorbehalten. " 111 Ob der Weg hin zu einer neuen Verfassung allerdings mit Art. 146 GG gangbar wäre, bleibt ebenfalls offen. ${ }^{112}$

Die Aussagen des Bundesverfassungsgerichts bleiben, soweit sie die zu erfolgende Verfassungsrevision bei Eintritt in einen europäischen Bundesstaat betreffen, eher im Ungewissen. ${ }^{113}$ Das ist nicht weiter verwunderlich, wenn man bedenkt, wie oben bereits kritisch angemerkt, ${ }^{114}$ dass der Programmsatz zur Gründung eines europäischen Bundesstaates weder im Vertrag von Lissabon angelegt ist, also nicht Entscheidungsgegenstand des Urteils war, noch derzeit erkennbare faktische Schritte auf politischer Ebene in diese Richtung unternommen werden. ${ }^{115}$ Es bleibt daher erneut Aufgabe der Rechtswissenschaft, die Andeutungen des Bundesverfassungsgerichts hinsichtlich des bereits lange und kontrovers diskutierten Themenkomplexes „Abwahl des Grundgesetzes“"116 in Bezug auf Ausgestaltung und Anforderungen an einen solchen Weg zu konkretisieren. Bereits die ersten Äußerungen nach Verkündung des Urteils in der Fachwelt zeigen,

108 BVerfGE 123, 267 ff., 332.

109 BVerfGE 123, 267 ff., 343.

110 BVerfGE 123, 267 ff., 349.

111 BVerfGE 123, 267 ff., 348.

112 Vgl. C. Tomuschat (Fn. 2), S. 272.

113 So auch inter alia: C. Hillgruber, in: V. Epping/C. Hillgruber, Beck'scher Online-Kommentar GG, 11. Edition 2011, Art. 146 GG, Rn. 11 a.E.; zu den Widersprüchlichkeiten der Ausführungen des Bundesverfassungsgerichts in dieser Hinsicht: J. Bröhmer, „Containment eines Leviathan“ - Anmerkungen zur Entscheidung des Bundesverfassungsgerichts zum Vertrag von Lissabon, in: ZEuS 2009, S. 543 ff., 552 ff.

114 Vgl. Fn. 32 und 66.

115 Vgl. etwa auf den gesamten Integrationsprozess abstellend M. Ruffert (Fn. 2), S. 1198; C. Schönberger (Fn. 24), S. 1209. Für den Vertrag von Lissabon selbst konstatiert dies sogar das Bundesverfassungsgericht, BVerfGE 123, 267 ff., 371.

116 Vgl. etwa die Darstellung bei M. Heckel, Die Legitimation des Grundgesetzes durch das deutsche Volk, in: HStR VIII, 1995, § 197, Rn. 1 ff. 
dass über den Weg zur Verfassungsneugebung in dieser Hinsicht weiterhin große Uneinigkeit herrscht. ${ }^{117}$

\section{Der Meinungsstreit um Art. 146 GG}

Die zur Beantwortung dieser Frage zunächst zu klärende Frage lautet demnach: Kann Art. 146 GG eine mögliche Grundlage zur Verfassungsneugebung durch den pouvoir constituant hin zu einem europäischen Bundesstaat sein? Dies hängt entscheidend davon ab, welches Verhältnis von Art. 146 GG zu Art. 79 Abs. 3 GG zugrunde gelegt wird. ${ }^{118}$

a) Wie bereits dargelegt, dekretiert das Bundesverfassungsgericht, dass nicht nur die in Art. 20 GG dargelegten Staatsprinzipien, sondern auch die souveräne Staatlichkeit der Bundesrepublik Deutschland selbst durch das Grundgesetz (Art. 79 Abs. 3 GG) garantiert sei und damit nicht durch ,einfache “119 Verfassungsänderung in den vorgesehenen Verfahren des Grundgesetzes, also durch den pouvoir constitué, beschränkt werden könnte. ${ }^{120}$ Über den Weg zu einer Betätigung des pouvoir constituant hin zu einer neuen Verfassung ist damit freilich noch nichts ausgesagt. ${ }^{121}$ Nur wenn auch Art. 146 GG sich an den Grenzen des Art. 79 Abs. 3 GG messen lassen müsste, wäre ein „Sich-auf-denWeg-machen“ hin zu einer neuen Verfassungsgebung innerhalb des geltenden Grundgesetzes ebenfalls für den pouvoir constituant ausgeschlossen. Wenn dem also wirklich so sein sollte, und es gibt jedenfalls in den Äußerungen des Präsidenten des Bundesverfassungsgerichts erneut Zweifel hieran, ${ }^{122}$ dann wäre eine Verfassungsgebung nicht mehr innerhalb rechtsstaatlicher Bahnen zu erlangen. Eine „Abwahl“ bzw. „Neugebung" fände nicht statt, sondern eine (aus rechtlicher Sicht) revolutionäre Lage müsste entstehen. ${ }^{123}$ Dass diese wohl in absehbarer Zeit für das Anliegen der Einführung eines „europäischen Bundesstaates“ nicht eintreten wird, dürfte auf der Hand liegen ${ }^{124}$ und war den beteiligten Richtern bei ihrer Urteilsfindung und Begründung sicher bewusst.

117 Vgl. K. F. Gärditz/C. Hillgruber (Fn. 2), S. 875; der dortigen Auffassung entgegentretend: D. Thym (Fn. 2), S. 566, dort Fn. 31. Die Problemlage der unterschiedlichen Deutungsmodelle aufzeigend: M. Kottmann/C. Wohlfahrt, (Fn. 2), S. $448 \mathrm{f}$.

118 J. Bröhmer (Fn. 113), S. 552 f.; C. Hillgruber (Fn. 113), Rn. 9.

119 Mit einer Zweidrittelmehrheit im Deutschen Bundestag und Bundesrat.

120 BVerfGE 123, 267 ff., 343; J. Bröhmer (Fn. 113), S. 552.

121 J. Bröhmer (Fn. 113), S. $552 \mathrm{f}$.

122 Vgl. Aussagen von Prof. Dr. Andreas Voßkuhle, zitiert in becklink 284799 vom 7.7.2009; vgl. auch Financial Times Deutschland vom 8.3.2011: „Voßkuhle hält europäischen Bundesstaat für möglich“, abrufbar unter: http://www.ftd.de/politik/europa/:vereinigte-staatenvon-europa-vosskuhle-haelt-europaeischen-bundesstaat-fuer-moeglich/60022927.html.

123 Inter alia H. Dreier, in: ders. (Hrsg.), GG-Kommentar, Band II, 2. Auflage 2006, Art. 79 Abs. 3 GG, Rn. 14 ff.; M. Hahn, Mehr Demokratie wagen: „Lissabon“-Entscheidung und Volkssouveränität, in: ZEuS 2009, S. 583 ff. 595; C. Tomuschat (Fn. 2), S. 272 f.; M. Herdegen, in: T. Maunz/G. Dürig, GG-Kommentar, 61. Ergl. 2011, Art. 79 GG, Rn. 75.

124 Daher auch der überlieferte Einwand von E.-W. Böckenförde: „Das ist so hoch gehängt, da kommt man nicht ran.“, zitiert in becklink 284799 vom 7.7.2009. In diese Richtung auch C. Calliess (Fn. 60), S. $574 \mathrm{f}$. 
b) Ist also verfassungsrechtlich eine Bindung von Art. 146 GG an Art. 79 Abs. 3 GG geboten? Hierzu ist zunächst ein Blick auf die Entstehungsgeschichte der Grundgesetznorm des Art. 146 GG in der heutigen Fassung zu werfen. Aufgrund der Aufteilung Deutschlands in Besatzungszonen nach dem Ende des Zweiten Weltkrieges wurde im Zuge der Verfassungsberatungen die Besonderheit des Art. 146 a.F. GG in das Grundgesetz aufgenommen. Der Wortlaut des ,alten“ Art. 146 GG, der im Zuge der Wiedervereinigung abgeändert wurde, diente vornehmlich dazu, nach Erfüllung des Wiedervereinigungsauftrages gemäß der damaligen Präambel des deutschen Grundgesetzes eine Verfassung mittels einer gesamtdeutschen Abstimmung durch die verfassungsgebende Gewalt zu wählen. ${ }^{125}$ Einer Bindung von Art. 146 GG an die Ewigkeitsgarantie des Art. 79 Abs. 3 GG, ebenso wie einer Obsoletwerdung nach Erfüllung des Wiedervereinigungsauftrages durch den Beitritt der ehemaligen DDR zur BRD wurde im Parlamentarischen Rat jedoch eine Absage erteilt. ${ }^{126}$ Art. 146 n.F. GG ist auf der alten Fassung des Artikels aufgebaut und wurde lediglich durch eine klärende kleinere Einfügung (Dieses Grundgesetz, das nach Vollendung der Einheit und Freiheit Deutschlands für das gesamte Deutsche Volk gilt, [...]; [eigene kursive Hervorhebung der Einfügung]) abgeändert. ${ }^{127}$ Man kann von der Entstehungsgeschichte des Art. 146 GG her diesen folglich nach wie vor als eine Verfassungsnorm ansehen, die eine legale Ablösung des Grundgesetzes durch Betätigung und Äußerung des Willens des pouvoir constituant zugunsten etwa eines europäischen Bundesstaates ermöglicht und dabei nicht von Art. 79 Abs. 3 GG begrenzt ist. ${ }^{128}$ Eine andere, wohl herrschende Sichtweise geht jedoch davon aus, dass durch den Beitritt der ehemaligen DDR zur BRD gemäß Art. 23 a.F. GG Art. 146 GG seine Berechtigung und Wirkung verloren habe. ${ }^{129}$ Die entscheidende Weichenstellung für die Beantwortung der Frage, ob Art. 146 GG auch für die verfassungsgebende Gewalt noch Anwendung finden oder heute allenfalls bei einer Verfassungsänderung noch erheblich sein kann, ist die Entscheidung, ob man der Kontinuitätsthese oder der Konsumtionsthese folgt. Nach der Kontinuitätsthese ${ }^{130}$ bzw. Perpe-

125 H. Dreier, in: ders. (Hrsg.), GG-Kommentar, Band III, 2. Auflage 2008, Art. 146 GG, Rn. $1 \mathrm{ff}$;; zur Entwicklungsgeschichte allgemein siehe etwa auch: H. Hofmann, in: B. Schmidt-Bleibtreu/H. Hofmann/A. Hopfauf, GG-Kommentar, 12. Auflage 2011, Art. 146 GG, Rn. 1 ff.

126 H. Dreier (Fn. 125), Rn. 9; a.A. J. Isensee, Schlussbestimmung des Grundgesetzes: Artikel 146, in: HStR VII, 1992, §166, Rn. $34 \mathrm{f}$.

127 H. Dreier (Fn. 125), Rn. 11.

128 H. Dreier (Fn. 125), Rn. 16; ders. (Fn. 123), Rn. 57: Art. 79 Abs. 3 GG werde durch eine Eingliederung in einen europäischen Bundesstaat transzendiert, da Art. 79 Abs. 3 GG nur auf eine Verfassungsänderung abstelle, die Eingliederung in einen europäischen Bundesstaat sei aber von grundsätzlicherer Qualität und müsse durch Betätigung des Willens der verfassungsgebenden Gewalt zum Ausdruck gebracht werden, für diese Betätigung stehe von Grundgesetz wegen Art. 146 GG zur Verfügung; ebenso: H. D. Jarass, in: ders./B. Pieroth, GG-Kommentar, 11. Auflage 2011, Art. 146 GG, Rn. 5; bereits zum MaastrichtVertrag: D. Murswiek (Fn. 97), S. 188 f.; a.A. etwa S. Speckmaier, in: D. C. Umbach/T. Clemens, GG-Kommentar, Band II, 2002, Art. 146 GG, Rn. 19.

129 H. Dreier (Fn. 125), Rn. 29 m.w.N.

130 So A. v. Campenhausen/P. Unruh, in: H. v. Mangoldt/F. Klein/ C. Starck, GG-Kommentar, Band III, 6. Auflage 2010, Art. 146 GG, Rn. 6. 
tuierungstheorie ${ }^{131}$ gilt ein Teil des „alten“ Art. 146 GG im Art. 146 n.F. GG fort. Die Konsumtionsthese ${ }^{132}$ nimmt demgegenüber eine völlige Neuschöpfung des Art. 146 GG an. Daher erscheint es für die Beantwortung der aktuell durch das Lissabon-Urteil jedenfalls indirekt und theoretisch aufgeworfenen Frage der Neuverfassungsgebung als Voraussetzung für den Eintritt Deutschlands in einen europäischen Bundesstaat durch den unmittelbaren Willen des Deutschen Volkes aufschlussreich, die Argumentation der Rechtswissenschaft aus der Zeit der Wiedervereinigung ${ }^{133}$ heranzuziehen.

\section{3. Überlegungen zur weiteren Geltung des Art. 146 n.F. GG im Zuge der deutschen Wiedervereinigung}

Zur Zeit der deutschen Wiedervereinigung standen grundsätzlich zwei Wege zur Erreichung der Einheit Deutschlands offen. Zum einen der Beitritt über Art. 23 a.F. GG oder eine neue Verfassungsgebung gemäß Art. 146 a.F. GG. ${ }^{134}$ Gewählt wurde schließlich nach politisch geführten Auseinandersetzungen über den ,,besseren“ Weg zur Deutschen Einheit ${ }^{135}$ - der Weg des Beitritts gemäß Art. 23 a.F. GG. ${ }^{136}$ Umstritten bleibt bis zum heutigen Tag die Auswirkungen des Beitritts gemäß Art. 23 a.F. GG und der Neufassung des Art. 146 a.F. GG auf den Gehalt des Art. 146 n.F. GG. Viele Literaturstimmen sehen in dem Beitritt und der Neufassung des Art. 146 a.F. GG eine Novation (Konsumtionsthese), d.h. sie gehen von einer Diskontinuität ${ }^{137}$ der Art.146a.F. GG und Art. 146 n.F. GG aus. Dieser Ansicht liegt die Annahme zugrunde, dass sich Art. 23 a.F. GG und Art. 146 a.F. GG gegenseitig ausschlössen und durch Betätigung des einen zur Erreichung der deutschen Einheit der andere obsolet würde. ${ }^{138}$ Richtigerweise muss dieser Annahme jedoch entgegengetreten werden. ${ }^{139}$ Der Wortlaut des

131 J. Isensee (Fn. 126, Rn. 53 ff.), spricht von „Perpetuierungstheorie“ anstatt von Kontinuitätsthese, inhaltlich ergibt sich jedoch kein Unterschied.

132 A. v. Campenhausen/P. Unruh (Fn. 130), Rn. 6.

133 Die bedeutenden rechtswissenschaftlichen Fragestellungen in diesem Zusammenhang veranlasste die Vereinigung der Deutschen Staatsrechtlehrer zu einer Sondertagung am 27.4.1990 in Berlin; siehe VVDStRL 48 (1990).

134 Vgl. inter alia: J. Isensee, Staatseinheit und Verfassungskontinuität, in: VVDStRL 48 (1990), S. 40 ff., 46 f., 48 ff.; R. Scholz, in: T. Maunz/G. Dürig, GG-Kommentar, 61. Ergl. 2011, Art. 146 GG, Rn. 4 ff.

$135 \mathrm{Zu}$ den politischen Diskussionen um den Weg zur Deutschen Einheit siehe stellvertretend und prägnant: J. Isensee (Fn.126), Rn. 48 ff.; B. Stückrath, Art. 146 GG: Verfassungsablösung zwischen Legalität und Legitimität, 1997, S. 32 ff.; $110 \mathrm{ff}$.

136 Art. 1 EinigVtr.

137 So ausdrücklich J. Isensee (Fn. 126), Rn. 47.

138 So etwa: J. Isensee (Fn. 126), Rn. 47; ders. (Fn. 134), S. 53; R. Bartlsperger, Verfassung und verfassungsgebende Gewalt im vereinten Deutschland, in: DVBl. 1990, S. 1285 ff., 1292 ff.; C. Tomuschat, Wege zur deutschen Einheit, in: VVDStRL 49 (1990), S. 70 ff., 87; R. Scholz (Fn. 134), Rn. 1; M. Kirn, in: I. v. Münch/P. Kunig, GG-Kommentar, 4./5. Auflage 2003, Art. 146 GG, Rn. 4.

139 H. Dreier (Fn. 125), Rn. 31; M. Baldus, Eine vom deutschen Volk in freier Entscheidung beschlossene Verfassung - Zum Schicksal des Art. 146 GG nach Vorlage des Abschlussberichts der Gemeinsamen Verfassungskommission, in: KritV 76 (1993), 429 ff., 431 ff.; so jetzt auch unter Änderung der Ansicht zur Vorauflage: A. v. Campenhausen/P. Unruh (Fn. 130), Rn. 11. 
Art. 146 a.F. GG lautete: „Dieses Grundgesetz verliert seine Gültigkeit an dem Tag, an dem eine Verfassung in Kraft tritt, die von dem deutschen Volke in freier Entscheidung beschlossen worden ist." Einen Rekurs auf Art. 23 a.F. GG oder gar eine ausschließliche Bindung an eine Wiedervereinigung findet man, vom Wortlaut der Vorschrift ausgehend, auch bei Zusammenschau mit der damaligen Präambel des Grundgesetzes gerade nicht. ${ }^{140}$ Darüber hinaus ist bereits oben auf die geschichtliche Entwicklung der Norm hingewiesen worden, die neben der Option der Wiedervereinigung eben einen weitergehenden Gehalt intendierte: Die freie Entscheidung des deutschen Volkes über seine Verfassung. ${ }^{141}$ Treffend fasst dies Horst Dreier unter systematischen Gesichtspunkten zusammen, wenn er sagt: „Einheitsfrage und Verfassungsfrage waren zweierlei und blieben es." 142 Nimmt man - da auch weitere Auslegungsmethoden zu keinem anderen Ergebnis führen ${ }^{143}$ - zutreffend an, dass Art. 146 n.F. GG auf Art. 146 a.F. GG bezüglich der Ausübung der verfassungsgebenden Gewalt aufbaut und damit nicht durch die Wiedervereinigung obsolet werden konnte, so ist Art. 146 n.F. GG weder als ,verfassungswidriges Verfassungsrecht" ${ }^{144} \mathrm{zu}$ qualifizieren, da im Ergebnis keine dem Grundgesetz widersprechende neue Norm, sondern lediglich eine teilweise Fortgeltung der alten Norm durch die Neufassung bewirkt wurde, ${ }^{145}$ noch hat er sich durch „Zeitablauf“ nach der deutschen Wiedervereinigung, trotz entsprechenden Hinweises in Art. 5 Spiegelstrich 4 EinigVtr (,innerhalb von zwei Jahren“) und erfolgter Verfassungsrevision im Jahr $1994,{ }^{146}$ gleichsam erledigt. ${ }^{147}$ Denn für eine zeitliche Einschränkung bietet der „,neue“ Artikelwortlaut keinen Hinweis. In einer Schlussbestimmung wäre ein solcher, falls erwünscht, jedoch wohl ohne größere Probleme aufnehmbar gewesen. ${ }^{148}$

Gegen die hier vertretene und bestehende Auffassung der Perpetuierung des Art. 146 GG wird zum Teil eingewandt bzw. eingeschränkt, dass Art. 146 GG wohl mit einem gewissen Zeitablauf nach der Wiedervereinigung, in einem wie auch immer de-

140 H. Dreier (Fn. 125), Rn. 31; A. v. Campenhausen/P. Unruh (Fn. 130), Rn. 11; M. Sachs, Das Grundgesetz im vereinten Deutschland - endgültige Verfassung oder Dauerprovisorium?, in: JuS 1991, S. 985 ff., 990; für eine parallele Anwendung etwa: D. Grimm, Das Grundgesetz - eine Verfassung für das geeinte Deutschland?, in: KritV (1990), S. 148 ff., 155 f.; $P$. Häberle, Verfassungspolitik für die Freiheit und Einheit Deutschlands - Ein wissenschaftlicher Diskussionsbeitrag im Vormärz 1990, in: JZ 1990, S. 358 ff., 358, 359 ff.; a.A. K. Stern, Der verfassungsändernde Charakter des Einigungsvertrages, in: DtZ 1990, S. 289 ff., 293 f.; B. Kempen, Grundgesetz oder neue deutsche Verfassung?, in: NJW 1991, S. 964 ff., 966; R. Scholz (Fn. 134), Rn. 3 ff.

141 H. Dreier (Fn. 125), Rn. 31; M. Baldus (Fn. 139), S. 434.

142 H. Dreier (Fn. 125), Rn. 31 a.E.

143 Siehe die überzeugenden Ausführungen und Argumentationen in: H. Dreier (Fn. 125), Rn. 31 ff.; A. v. Campenhausen/P. Unruh (Fn. 130), Rn. 11 ff.; M. Baldus (Fn. 139), 432; ausführlich zudem B. Stückrath (Fn. 135), S. $97 \mathrm{ff}$.

144 So jedoch R. Bartlsperger (Fn. 138), S. 1301; a.A. B. Stückrath (Fn. 135), S. 110; E. Wiederin, Die Verfassungsgebung im wiedervereinten Deutschland - Versuch einer dogmatischen Zwischenbilanz zu Art. 146 GG n. F., in: AöR 117 (1992), S. 410 ff., 418 ff.

145 D. Heckmann, Gestaltungskraft und Geltungsverlust von Rechtsnormen - Elemente einer Theorie der autoritativen Normgeltungsbeendigung, 1997, S. $445 \mathrm{f}$.

146 Gesetz zur Änderung des Grundgesetzes vom 27.10.1994, BGBl. Teil I Nr. 75 vom 3.11.1994, S. 3146.

147 So jedoch etwa D. Heckmann (Fn. 145), S. 447 f.; M. Sachs (Fn. 140), S. 990.

148 H. Dreier (Fn. 125), Rn. 35 m.w.N. 
finierten Zeitkorridor, obsolet wurde. ${ }^{149}$ Diesem Einwand steht zum einen die Annahme der Anhänger der Konsumtionsthese nahe, dass gleichsam durch ,täglich angewandte Verfassungspraxis und -wirklichkeit“ bereits eine „Abstimmung“ über die Verfassung erfolgte und daher Art. 146 GG faktisch entleert wurde, ${ }^{150}$ zum anderen aber auch die Annahme, dass mit der Wiedervereinigung Art. 146 GG nur noch „,deklaratorischen Charakter" habe. ${ }^{151}$ Anzunehmen, eine derartige Grundsatzentscheidung, wie die der Verfassungsgebung, die Art. 146 GG seinem Wortlaut nach klar der freien Entscheidung des deutschen Volkes unterstellt, sei nicht durch klare Betätigung dieser freien Entscheidung, sondern gleichsam durch Passivität in Hinnahme der ,täglichen“ Grundgesetzanwendung umzusetzen, scheint dem Rang dieser Entscheidung kaum genügend Rechnung $\mathrm{zu}$ tragen. ${ }^{152} \mathrm{Zu}$ Recht ist ferner darauf hingewiesen worden, dass Art. 146 GG nicht nur rein deklaratorische Wirkung hat, sondern aufgrund der Perpetuierung weiterhin die Funktion erfüllt, ${ }^{153}$ eine ,Brücke“ ${ }^{\text {"154 }}$ zu einer freien Entscheidung des deutschen Volkes zu bauen, ohne hierbei auf revolutionäre Umbrüche setzen zu müssen. ${ }^{155}$

\section{Konsequenzen für einen möglichen Weg Richtung Bundesstaat „Europa“}

a) Legt man die hier vertretene Auffassung der Perpetuierung zugrunde, ist Art. 146 n.F. GG auch nach der deutschen Wiedervereinigung mit einem fortdauernden Gehalt unterlegt. Dieser ist mithin nicht im Zuge der Wiedervereinigung geändert worden und unterliegt daher auch nicht der Bindung an Art. 79 Abs. 3 GG. ${ }^{156}$ Denn es handelt sich bei Art. 146 GG durch die Perpetuierung nicht um eine derogative und nach-

149 Vgl. inter alia M. Sachs (Fn. 140), S. 990.

150 Wenn auch teilweise mit unterschiedlichen Begründungen und Herleitungen, so im Ergebnis inter alia: J. Isensee (Fn. 126), Rn. 36 ff.; R. Scholz (Fn. 134), Rn. 9; entschieden einer Entleerung entgegentretend etwa $H$. Meyer, Artikel 146 GG. Ein unerfüllter Verfassungsauftrag?, in: H. H. v. Arnim (Hrsg.), Direkte Demokratie - Beiträge auf dem 3. Speyerer Demokratieforum, Schriftenreihe der Hochschule Speyer Band 140, 2000, S. 67 ff., 79.

151 Vgl. inter alia: R. Zippelius, Quo vadis Grundgesetz?, in: NJW 1991, S. 23.

152 Im Zuge der Ausführungen des Bundesverfassungsgerichts im Lissabon-Urteil zum Selbstbestimmungsrecht des Deutschen Volkes (sic!) scheint diese Ansicht wohl auch mehr als angegriffen, wenn man denn überhaupt den Ausführungen des Gerichts zur Garantie der Staatlichkeit folgt und somit erst zu einer Erörterung des Art. 146 GG gelangt.

153 So jedoch ausdrücklich etwa R. Scholz (Fn. 134), Rn. 9.

154 H. Dreier (Fn. 125), Rn. 36.

155 H. Dreier (Fn. 125), Rn. 36; besonders anschaulich: H. Meyer (Fn. 150), S. 79; A. v. Campenhausen/P. Unruh (Fn. 130), Rn. 17; a.A. etwa U. di Fabio, Der neue Art. 23 Grundgesetz - Positivierung vollzogenen Verfassungswandels oder Verfassungsneuschöpfung?, in: Der Staat 32 (1992), S. 191 ff., 213.

156 H. Dreier (Fn. 125), Rn. 33; ebenso: C. Pestalozza, Neues Deutschland - in bester Verfassung?, in: Jura 1994, S. 561 ff., 566; H. Meyer (Fn. 150), S. 79 f.; E. Wiederin (Fn. 144), S. 432; M. Baldus (Fn. 139), S. 432 f.; a.A. etwa K. F. Gärditz/C. Hillgruber (Fn. 2), S. 875 f.; S. Speckmaier (Fn. 128), Rn. 19; H. Weis, Verfassungsrechtliche Fragen im Zusammenhang mit der Herstellung der staatlichen Einheit Deutschlands, in: AöR 116 (1991), S. 1 ff., 30: Ein Verfassungsentwurf wäre an Art. 79 Abs. 3 GG gebunden; H.-U. Erichsen, Die Verfassungsänderung nach Art. 79 GG und der Verfassungsbeschluß nach Art. 146 GG, in: Jura 1992, S. 52 ff., 55 a.E. 
konstitutionelle, ${ }^{157}$ sondern um eine fortgeltende Norm, die dem pouvoir constituant eine grundgesetzkonforme Möglichkeit der Verfassungsgebung eröffnet. ${ }^{158}$ Es geht in Art. 146 GG nicht um eine Verfassungsänderung durch den pouvoir constitué, die den Vorgaben des Art. 79 GG unterliegt, sondern um eine Verfassungsgebung durch den pouvoir constituant. Diese ist den Bindungen des Art. 79 GG entzogen. ${ }^{159}$

b) Das Lissabon-Urteil lässt die Frage, ob der pouvoir constituant an Art. 79 Abs. 3 GG gebunden ist, wie bereits erwähnt, explizit offen. ${ }^{160}$ Bei einer Bindungswirkung bliebe letztlich nur der Weg einer Revolution. ${ }^{161}$ Es ist in der Besprechungsliteratur bereits darauf verwiesen worden, dass ein Fingerzeig des Bundesverfassungsgerichts auf diesen Weg von einem Verfassungsgericht, das sich selbst als „Hüter der Verfassung“ sieht, jedenfalls irritierend wirken würde. ${ }^{162}$ Einen Modus für ein Verfahren nach Art. 146 GG legt das Gericht jedenfalls nicht fest. ${ }^{163}$

c) Ist also Art. 146 GG richtigerweise nicht von Art. 79 GG beschränkt, stellt sich die Frage, wie eine Aktivierung des pouvior constituant in realiter bewerkstelligt werden könnte. Konkrete Vorgaben über den Modus einer solchen Aktivierung und eine daraufhin folgende Entscheidung beinhaltet Art. 146 GG nicht. ${ }^{164}$ Der Wortlaut spricht lediglich von der „freien Entscheidung“ durch das deutsche Volk. Dies bestätigt zunächst nur die hier vertretene Ansicht, dass eine Bindung an Art. 79 GG nicht besteht und die Initiative zu einer neuen Verfassungsgebung grundsätzlich vom Volk ausgehen muss, es bedarf mithin einer Verfassungsinitiative aus einer Volksbewegung heraus oder durch die das Volk vertretenden Organe auf Bundesebene. ${ }^{165} \mathrm{Ob}$ die aus dieser Initiative sich heraus entwickelnde Verfassungsneugebung am Ende durch eine Volksabstimmung oder durch eine verfassungsgebende Nationalversammlung, die zuvor durch den pouvior constituant gewählt wurde, erfolgen kann, geht daraus jedoch noch nicht hervor. ${ }^{166}$ Entgegen mancher Stimmen in der Literatur ${ }^{167}$ erscheint es aufgrund des weiteren Wortlauts allerdings vorzugswürdig, dass ein solcher grundsätzlicher Akt der Annahme

157 So jedoch inter alia K. F. Gärditz/C. Hillgruber (Fn. 2), S. 875 f.; R. Bartlsperger (Fn. 138), S. 1298 f.; J. Isensee (Fn. 126), Rn. 61.

158 H. Dreier (Fn. 125), Rn. 33; A. v. Campenhausen/P. Unruh (Fn. 130), Rn. 17 ff.

159 Vgl. Nachweise in Fn. 156.

160 BVerfGE 123, 267 ff., 343.

161 Siehe inter alia J. Bröhmer (Fn. 113), S. 553; C. Calliess (Fn. 60), S. 575; D. Halberstam/ C. Möllers (Fn. 2), S. 1256.

162 M. Hahn (Fn. 123), S. 595.

163 D. Thym (Fn. 2), S. 565.

164 A. v. Campenhausen/P. Unruh (Fn. 130), Rn. 23 f.; K. Stern (Fn. 140), S. 293; ausführlich zu einem möglichen Verfahrensgang etwa: H. Moelle, Der Verfassungsbeschluß nach Artikel 146 Grundgesetz, 1996, S. $197 \mathrm{ff}$.

165 Letzteres ist erneut umstritten. Eine Aktivierung des Volkswillens auch durch Bundesorgane für möglich erachtend etwa A. v. Campenhausen/P. Unruh (Fn. 130), Rn. 23; B. Stückrath (Fn. 135), S. 112 ff., 154 f.; D. Thym (Fn. 2), S. 566; dem entgegentretend, da eine Bindung des Art. 146 GG an Art. 79 Abs. 3 GG annehmend etwa: K. F. Gärditz/C. Hillgruber (Fn. 2), S. 876.

166 So auch H. Dreier (Fn. 125), Rn. 52; A. v. Campenhausen/P. Unruh (Fn. 130), Rn. 23.

167 Beide Wege als möglich erachtend etwa: H. Dreier (Fn. 125), Rn. 52; H. D. Jarass (Fn. 128), Rn. 3. 
der neuen Verfassung am Ende eines Verfahrens nach Art. 146 GG nicht durch eine verfassungsgebende Nationalversammlung, sondern lediglich durch eine Volksabstimmung erfolgen kann. ${ }^{168}$ Nimmt man Bezug auf das Lissabon-Urteil und die Insistenz des Gerichts bezüglich der freien Selbstbestimmung der verfassungsgebenden Gewalt, so scheint diese Betrachtungsweise des Art. 146 GG jedenfalls diejenige zu sein, die der Forderung nach einer aktiven Rolle des pouvoir constituant am ehesten entspräche. Sollte der Weg über eine Volksabstimmung erfolgen, kann jedoch Art. 146 GG nicht als Grundlage einer solchen Volksabstimmung herangezogen werden, denn er beinhaltet nur die grundgesetzkonforme Möglichkeit der Verfassungsgebung, die entsprechenden Modalitäten legt er nicht fest. ${ }^{169}$ Einer Volksabstimmung zur Verfassungsgebung müsste daher eine Grundgesetzänderung zur Ermöglichung dieser Volksabstimmung - zumindest in dieser Frage ${ }^{170}$ - vorausgehen. ${ }^{171}$ Da einer Volksabstimmung ein konkreter Entwurf zugrunde gelegt werden müsste, ${ }^{172}$ wäre neben der Schaffung der Möglichkeit einer Volksabstimmung im Grundgesetz zudem ein Verfassungskonvent vom deutschen Volk zu wählen, der dann einen entsprechenden Entwurf ggf. unter Beteiligung interessierter Parteien ausarbeitet, diesen jedoch nicht selbst für das Volk verabschiedet, sondern dem deutschen Volk gesamt zur Abstimmung stellt. ${ }^{173}$ Eine bestimmte Anforderung an ein Quorum für die letztlich erfolgende Abstimmung über die Verfassung kann dem Wortlaut des Art. 146 GG hierfür nicht entnommen werden. ${ }^{174}$ Da der pouvoir constituant jedoch nicht an Vorgaben des Art. 79 GG gebunden ist, würde das deutsche Volk nach allgemeinen Gesichtspunkten des Demokratieprinzips mit seiner einfachen Mehrheit ${ }^{175}$ eine neue Verfassung durch Beschluss bestimmen. Der Einwand, dass dies im Vergleich zu einer derzeit möglichen „einfachen“ Verfassungsänderung durch den pouvoir constitué, der einer Zweidrittelmehrheit unterliegt (Art. 79 Abs. 2 GG), eine zu geringe Schwelle sei, ${ }^{176}$ greift nicht. Denn nicht der vertretene Wille des Volkes be-

168 Jedenfalls für die Entscheidung des „ob“ einer Verfassungsneugebung: P. M. Huber, in: M. Sachs (Hrsg.), GG-Kommentar, 5. Auflage 2009, Art. 146 GG, Rn. 16 f., der jedoch für eine „Endabstimmung“" auch eine Nationalversammlung für ggf. entscheidungsbefugt hält; ferner: J. Wolf, Die Revision des Grundgesetzes durch Maastricht - Ein Anwendungsfall des Art. 146 GG, in: JZ 1993, S. 594 ff., 600, der bereits damals eine Verletzung des Art. 146 GG als individualverfassungsbeschwerdefähig angesehen bzw. dessen Implementation gefordert hat.

169 M. Heckel (Fn. 116), Rn. 92; M. Kirn (Fn. 138), Rn. 8.

170 Zur grundsätzlichen Möglichkeit der Einführung plebiszitärer Elemente in das Grundgesetz siehe etwa: P. Krause, Verfassungsrechtliche Möglichkeiten unmittelbarer Demokratie, in: HStR III, 2005, § 35, Rn. 1 ff.; I. Ebsen, Abstimmungen des Bundesvolkes als Verfassungsproblem, in: AöR 110 (1985), S. $2 \mathrm{ff}$.

171 So überzeugend P. M. Huber (Fn. 168), Rn. 16; ein einfaches Durchführungsgesetz für ausreichend erachtend etwa: M. Baldus (Fn. 139), S. 435.

172 Vgl. etwa H. Moelle (Fn. 164), S. 57; H. D. Jarass (Fn. 128), Rn. 4; S. Speckmaier (Fn. 128), Rn. $16 \mathrm{f}$.

173 So auch J. Wolf (Fn. 168), S. 600; diesen Weg als möglich ansehend: H. Dreier (Fn. 125), Rn. 52.

174 H. Dreier (Fn. 125), Rn. 53.

175 So auch H. Moelle (Fn. 164), S. 60 f., 197 ff.; H. D. Jarass (Fn. 128), Rn. 4; S. Speckmaier (Fn. 128), Rn. 28, allerdings von einer Novation in Art. 146 GG ausgehend.

176 H. Moelle (Fn. 164), S. 60 f., 210 ff., der dort auch den Einwand einer zwingenden Zweidrittelmehrheit behandelt. 
schließt etwas, sondern das Volk selbst, das eben nicht an Art. 79 GG gebunden ist, stimmt ab; mithin liegt eine direktere Legitimation zugrunde, für die eine „einfache“ Mehrheit des gesamten Volkes ausreicht. ${ }^{177}$

d) Unter den vorgenannten Prämissen wäre demnach eine Volksabstimmung der verfassungsgebenden Gewalt in den Bahnen des deutschen Grundgesetzes gemäß Art. 146 GG i.V.m. einer zu schaffenden Rechtsgrundlage für eine solche Volksabstimmung über einen europäischen Bundesstaat möglich. Einer Revolution in Deutschland bedürfte es nicht. ${ }^{178}$

\section{Es bleibt beim „Eventuell“}

Zusammenfassend lässt sich demnach feststellen, dass das Bundesverfassungsgericht aus seiner etatischen Sicht der Dinge die Grenze einer Integration Deutschlands in einen - nicht in der zu entscheidenden Vertragslage vorgesehenen und darüber hinaus auch politisch derzeit nicht angedachten - ,europäischen Bundesstaat“" im Zuge der gleichzeitig festgelegten Integrationspflicht Deutschlands vorgegeben hat. Die Ausführungen, die letztlich zu einer Garantie der Staatlichkeit führen, überzeugen nicht. Mit seinem viel besprochenen Urteilsspruch ${ }^{179}$ hat das Bundesverfassungsgericht die Tür zu einer Weiterentwicklung Europas auch zu einem Bundesstaat allerdings nicht völlig versiegelt, sondern die Entwicklung nur sehr erschwert. ${ }^{180}$ Wenn man denn überhaupt in den Grenzen des Nationalstaats- und des Bundesstaatsmodells bleibt, wäre als Endstufe der Integration die europäische Einheit im Bundesstaat denkbar. Auf den Weg hin zu dieser Einheit könnte sich das deutsche Volk bei richtigem Verständnis von Art. 146 GG auch auf dem Boden des Grundgesetzes aufmachen. Das „Odium des Verfassungsbruches" ${ }^{\text {" } 181}$ wäre damit nicht verbunden. Bei aller Kritik, die auch hier geäußert wurde, ist dem Bundesverfassungsgericht jedenfalls zu verdanken, dass ganz klar eine Zäsur erfolgen müsste, um in einen europäischen Bundesstaat einzutreten; die Bürgerinnen und Bürger Deutschlands sich also klar zu einem solchen Modell der europäischen Einigung bekennen müssten. ${ }^{182}$ Dieser Dank bezieht sich, sollte ein europäischer Bundesstaat überhaupt jemals zur Debatte stehen, vor allem darauf, dass damit eine eventuelle Diskrepanz zwischen Regierten und Regierenden in Fragen der europäischen Integration überwunden werden könnte. Rechtlich ist die Herleitung des Bundesverfassungsgerichts nicht zwingend. Der oft zitierte Traum politischer Größen der Nachkriegsjah$\mathrm{re}^{183}$ ist nicht ausgeträumt. $\mathrm{Ob}$ die Erfüllung aller Träume wünschenswert ist, ist eine

177 So auch H. Moelle (Fn. 164), S. 60 f., $210 \mathrm{ff}$.

178 So auch inter alia F. Schorkopf (Fn. 62), S. 723 f.

179 Letztlich erhebt auch das Bundesverfassungsgericht einen gewissen Anspruch auf diese mediale Präsenz, wenn es das Urteil auch in englischer Sprache online stellt, wohl um als Vorbild auch ausländischer Rechtsprechung gleichsam zu fungieren.

180 Vgl. inter alia den Einwand Böckenfördes (Fn. 124); M. Selmayer (Fn. 4), S. 646.

181 R. Zippelius (Fn. 151), S. 23.

182 In diese Richtung auch: F. Schorkopf (Fn. 2), S. 1239; ders. (Fn. 62), S. 724; D. Grimm (Fn. 2), S. 490.

183 Vgl. zur häufig zu findenden Aufzählung etwa M. Selmayer (Fn. 4), S. 644. 
Frage, die von der Möglichkeit ihrer Erfüllung zu trennen ist. Die derzeitigen Umtriebe auf europäischer Ebene in Sachen Weltwirtschaft und Währungsstabilität lassen aus diesem Traum vielleicht eher einen Albtraum werden. Nichtsdestotrotz zeigen gerade solche Extremsituationen, wie schnell Traum und Realität unter Umständen zusammenkommen. Eine rechtswissenschaftliche Auseinandersetzung, die diese Aspekte unter Einbeziehung des seit über zwanzig Jahren vorhandenen Meinungsstreits in Bezug auf Art. 146 GG anhand des Lissabon-Urteils erneut aufgreift, ist daher nötig.

Nach hier vertretener Ansicht bräuchte es zwar den Gang über Art. 146 GG - der jedoch möglich und von den Bindungen des Art. 79 Abs. 3 GG nicht berührt wäre nicht, da nur Staatsstrukturprinzipien, aber eben nicht der Staat in seiner heutigen bundesdeutschen Gestalt als solcher über Art. 79 Abs. 3 GG geschützt ist. ${ }^{184}$ Da aber das Bundesverfassungsgericht anders dekretiert hat, müsste dieser Weg notfalls beschritten werden. Dazu bräuchte es freilich mutiger Handlungsbereitschaft. Sicherlich wird sich dann das Bundesverfassungsgericht erneut mit diesem Themenkomplex auseinandersetzen müssen und seine Antwort auf die dann auch ihm gestellte Frage geben, ob der pouvoir constituant - wie und warum auch immer - an bestimmte Vorgaben gebunden sein soll. ${ }^{185}$ Denn das Bundesverfassungsgericht behält sich die Prüfung des Weges über Art. 146 GG vor und erwartet augenscheinlich eine ihm angetragene Prüfung in dieser Frage für die Zukunft. ${ }^{186}$

184 Siehe Nachweise Fn. 94.

185 Für eine Interpretation, die bereits jetzt Anlagen für einen solchen Weg durch die Ausführungen im Lissabon-Urteil sieht, bietet das umfangreiche Urteil in dieser Hinsicht m.E. keine stichhaltigen Ansichtspunkte.

186 BVerfGE 123, 267 ff., 332; inter alia F. Schorkopf, (Fn. 2), S. 1238; D. Halberstam/C. Möllers (Fn. 2), S. 1256; D. Thym (Fn. 2), S. 565; E. Pache (Fn. 24), S. 287. 